\title{
Dark Energy in the Universe, the Irreversibility of Time and Neutrinos
}

\author{
N. E. Mavromatos \\ King's College London, Department of Physics, Theoretical Physics, Strand, London WC2R 2LS, U.K. \\ Received on 11 December, 2004
}

\begin{abstract}
In this review, I discuss briefly how the presence of a cosmological constant in the Universe may imply a decoherent evolution of quantum matter in it, and as a consequence a fundamental irreversibility of time unrelated in principle to CP properties (Cosmological CPT Violation). In this context, I also discuss recently suggested novel possible contributions of massive neutrinos to the cosmological constant, which are not due to the standard loop expansion in quantum field theory, but rather due to unconventional properties of (some version of) the quantum theory underlying flavour mixing. It is also argued that quantum space time foam may be responsible for the neutrino mass differences, observed today, and through the above considerations, for the (majority of the) dark energy of the Universe in the present era. In the above context, I also present a fit of all the currently available neutrino oscillation data, including the LSND "anomalous" experimental results, based on such a CPT Violating decoherent neutrino model. The key feature is to use different decoherent parameters between neutrinos and antineutrinos, due to the above-mentioned CPT violation. This points to the necessity of future experiments, concentrating on the antineutrino sector, in order to falsify the model.
\end{abstract}

\section{INTRODUCTION}

Recent astrophysical observations, using different experiments and diverse techniques, seem to indicate that $70 \%$ of the Universe energy budget is occupied by "vacuum" energy density of unknown origin, termed Dark Energy [1,2]. Best fit models give the positive cosmological constant EinsteinFriedman Universe as a good candidate to explain these observations, although models with relaxing to zero vacuum energy (quintessential, i.e. involving a scalar field which has not yet reached the minimum of its potential) are compatible with the current data.

From a theoretical point of view the two categories of Dark Energy models are quite different. If there is a relaxing to zero cosmological vacuum energy, depending on the details of the relaxation rate, it is possible in general to define asymptotic states and hence a proper Scattering matrix (S-matrix) for the theory, which can thus be quantised canonically. On the other hand, Universes with a cosmological constant $\Lambda>0$ (de Sitter) admit no asymptotic states, as a result of the Hubble horizon which characterises these models, and hampers the definition of proper asymptotic state vectors, and hence a proper S-matrix. Indeed, de Sitter Universes will expand for ever, and eventually their constant vacuum energy density component will dominate over matter in such a way that the Universe will enter again an exponential (inflationary) phase of (eternal) accelerated expansion, with a Hubble horizon of radius $\delta_{H} \propto 1 / \sqrt{\Lambda}$. It seems that the recent astrophysical observations $[1,2]$ seem to indicate that the current era of the universe is the beginning of such an accelerated expansion.

Canonical quantisation of field theories in de Sitter space times is still an elusive subject, mostly due to the abovementioned problem of a proper S-matrix definition. One suggestion towards the quantisation of such systems could be through analogies with open systems in quantum mechanics, interacting with an environment. The environment in cosmological constant models would consist of field modes whose wavelength is shorter than the Hubble horizon radius. This splitting was originally suggested by Starobinski [3], in the context of his stochastic inflationary model, and later on was adopted by several groups [4]. Crossing the horizon in either direction would constitute interactions with the environment. An initially pure quantum state in such Universes/opensystems would therefore become eventually mixed, as a result of interactions with the environmental modes, whose strength will be controlled by the size of the Hubble horizon, and hence the cosmological constant. In particular, for some simple cases, such as conformally coupled scalar fields [4] in de Sitter spaces it has been shown explicitly that the system modes decohere if they have wavelengths longer than a critical value, which is of the order of the Hubble horizon. Such decoherent evolution could explain the classicality of the early (or late, in the case of a cosmological constant) Universe phase transitions [5]. The approach is still far from being complete, not only due to the technical complications, which force the researchers to adopt severe, and often unphysical, approximations, but also due to conceptual issues, most of which are associated with the back reaction of matter onto space time, an issue often ignored in such a context. It is my opinion that the latter issue plays an important rôle in the evolution of a quantum Universe, especially one with a cosmological constant, and is associated with quantum gravity issues. The very origin of the cosmological constant, or in general the dark energy of the vacuum, is certainly a property of quantum gravity.

Since string theory seems to be the most rigorous and most successful approach to quantum gravity, to date, encompassing the known quantum field theories of flat space times in its low energy limit, I would like to approach the problem of the cosmological constant in this framework. Critical string theory models, which are based on S-matrix theory, at least for their perturbative formulation, can only accommodate relaxing to zero vacuum energy models, which allow for a proper definition of asymptotic states, but cannot deal with de Sitter Universes [6]. On the other hand, string/membrane theory models with anti-de-Sitter backgrounds, which admit supersymmetry, are consistent. In critical string theory an evolution of pure states to mixed do not exist, and this is another way of understanding the incompatibility of conventional strings with de Sitter Universes. However, quantum effects in strings, generated by dilaton tadpoles in $\sigma$-models 
formulated in world sheets with higher topologies (genera) lead to non-zero contributions to the cosmological constant (Fischler-Susskind mechanism) [7]. Modular divergences in such theories require regularisation, which essentially means that small handles in closed strings (appropriate for gravity), of size smaller than the world-sheet short-distance cutoff, must be integrated out in an effective Wilsonian path integral, since such small handles could not be distinguished from tree-level world-sheet topologies. This corresponds to adding to the tree level $\sigma$-model action a world-sheet counterterm appropriate for absorbing such modular (small handle) divergences. The effects of such terms lead to corrections in the tree-level graviton world-sheet $\beta$-functions similar to those arising in a de Sitter Universe. However, the issue of the impossibility of defining a proper S-matrix in de Sitter Universe brings up an immediate question on the consistency of the approach within string theory.

\section{NON-CRITICAL STRINGS IN DE SITTER BACKGROUNDS}

Fortunately there is a way out, which goes beyond the above-described Fischler-Susskind mechanism for generating cosmological constant contributions. From a worldsheet view point, a non-zero cosmological constant amounts to contributions to the effective central charge of the twodimensional world-sheet field theory, which thus deviates from its (conformal point) critical value. Stringy $\sigma$-models with a non-zero central charge deficit constitute the so-called Liouville strings [8]. The path-integral world-sheet quantisation of such non-critical strings requires the introduction of the Liouville mode $\phi$, which is an extra world-sheet field whose target-space signature (time-like or space-like) depends on the signature of the central charge deficit (positive or negative respectively). This extra field, plays the rôle of a new coordinate in target space, and its presence is responsible for the restoration of the conformal invariance of the theory [8].

An important step towards the physical significance of the Liouville mode is the identification [9] of its world-sheet zero mode $\phi_{0}$ with the target time, in the supercritical (positive central charge deficit) theories. Such an identification emerges from dynamics of the target space low-energy effective field theory [10], in the sense of minimisation of the effective potential. Furthermore it can be shown rigorously [9] that under such an identification one cannot define a pure-state S-matrix but rather a $\$$-matrix, which is a non-factorisable product of $S$ and $S^{\dagger}$, acting on density matrix mixed states rather than pure states. The non factorisability may be attributed to divergences in the short-distance world-sheet behaviour of the $\sigma$-model theory.

With this identification in mind, one may proceed to discuss the issue of propagation of quantum matter in a de Sitter Background, within such a non-critical string framework. By following simple arguments on world-sheet renormalisationgroup invariance of $\sigma$-model quantities which have targetspace physical relevance, it is straightforward to arrive at the following master equation describing the evolution of string low-energy matter in a non-conformal $\sigma$-model background:

$$
\dot{\rho}=i[\rho, H]+: \beta^{i} \mathcal{G}_{i j}\left[g^{j}, \rho\right]:
$$

where $\rho$ is the density matrix of string matter excitations, $H$ is the effective low-energy matter Hamiltonian, $g^{i}$ are background target-space fields, and $\beta^{i}$ are their corresponding $\sigma$-model renormalisation group $\beta$-functions, expressing their scaling under Liouville dressing [8]. Canonical quantisation for the operators/fields $g^{i}$ is possible in Liouville strings [9], as a result of summing up higher world-sheet topologies, and thus : $\cdots:$ in (1) denotes appropriate quantum ordering. The quantity $G_{i j}=2 z^{2} \bar{z}^{2}<V_{i}(z) V_{j}(0)>$ is the so-called Zamolodchikov "metric" in the moduli space of the string, a two-point correlation function with respect to the vertex operators $V_{i}$ corresponding to the deformations of the $\sigma$-model action from the conformal point $S^{*}$ :

$$
S_{\sigma}=S^{*}=g^{i} \int_{\Sigma} V_{i}
$$

where $\int_{\Sigma}$ denotes integration over the world-sheet. The dot over $\rho$ in (1) denotes differentiation with respect to the worldsheet zero mode of the Liouville field, identified in this approach with the target time [9]. An important note for the compact notation in (2) is now in order. The index $i$ runs over both species of background target space fields as well as space-time coordinates. Thus the summation over $i, j$ indices in (1) corresponds to a summation over $M, N$ indices but also a continuous generally covariant space-time integration $\int d^{d} y \sqrt{-g}$, where $y$ denotes a set of d-dimensional space-time coordinates. It is important to stress that strings respect general covariance by construction. For instance, for the case at hand, where we are interested in perturbations of the metric background $g_{M N}$, where $M, N$ are target space-time indices, one has the correspondence:

$$
\begin{aligned}
g^{i} & \rightarrow g_{M N}(y), \\
V_{i} & \rightarrow V^{M N}(X, y)=\partial_{\alpha} X^{M} \partial^{\alpha} X^{N} \delta^{(d)}(y-X(\sigma, \tau)), \\
\text { where } \alpha & =\sigma, \tau \\
\int_{\Sigma} g^{i} V_{i} & \rightarrow \int d^{d} y \sqrt{-g} g_{M N}(y) \int_{\Sigma} V^{M N}(X, y)
\end{aligned}
$$

For conformal world-sheet backgrounds $\beta^{i}=0$ and one obtains a normal quantum mechanical equation where purity of states is preserved under evolution. When nonconformal string backgrounds are present, however, one has non-quantum mechanical corrections terms in this evolution, which in general may imply decoherence of matter, that is evolution of initially pure states to mixed ones. In a perturbative derivative expansion (in powers of $\alpha^{\prime}$, where $\alpha^{\prime}=\ell_{s}^{2}$ is the Regge slope of the string, and $\ell_{s}$ the fundamental string length), the lowest order graviton $\beta$ function is just the Ricci tensor

$$
\beta_{M N}=\alpha^{\prime} R_{M N}+\ldots
$$

where the ... indicate terms higher order in $\alpha^{\prime}$, which can be ignored in a low-energy (infrared) framework for the target 
space effective field theory, we are interested in here. From now on, unless otherwise stated, we shall work in units of $\alpha^{\prime}=1$ for brevity.

Conformal backgrounds in string theory are therefore Ricci flat backgrounds in this framework. On the other hand, de Sitter backgrounds, for which $R_{M N} \propto \Lambda g_{M N} \neq 0$, with $\Lambda>0$ a cosmological constant, obviously violate this condition, and the excitation of strings in such backgrounds can be described, at least perturbatively for the (physically relevant) case of small $\Lambda$, so that the deviation from the Ricci flatness is minute, by means of a non-critical Liouville dressed $\sigma$-model. From (1), (3), the evolution equation of low-energy string matter in such a background, then, reads in this case [11]:

$$
\dot{\rho}=i[\rho, H]+\int d^{d} y \Lambda: \sqrt{-g} g_{M N}\left[g^{M N}, \rho\right]:
$$

where we took into account the fact that to lowest order, the Zamolodchikov metric is just the appropriate tensorial identity in all sets of indices ( $\mathrm{M}$ and $y$ ). If one chooses an antisymmetric ordering prescription, and adopts a weak-graviton expansion about flat Minkowski space time, $g_{M N}=\eta_{M N}+h_{M N}$, which seems to be the cosmologically relevant case for the present era of the Universe, then one arrives at a double commutator structure for the decoherence term [11]:

$$
\dot{\rho}=i[\rho, H]+\int d^{d} y \Lambda\left[h_{M N},\left[h^{M N}, \rho\right]\right]
$$

Notice that the decoherence term, which is real, is not invariant, due to its structure, under the time reversal symmetry $t \rightarrow-t$ (we remind the reader that under such a symmetry, the matter Hamiltonian is time reversal invariant, but the $i \rightarrow-i$. Moreover, since we are dealing with small perturbations around flat Minkowski space time, the quanta of the gravitational field $h_{M N}$ can be taken to respect the time reversal symmetry). This breaking of the time reversal invariance is unrelated in principle to properties of matter under the discrete symmetries of Charge $(\mathrm{C})$ and Parity $(\mathrm{P})$, and thus the $\Lambda$-induced decoherence term is CPT violating. This is what I would call Cosmological CPT Violation [11], due to the global nature of the non-quantum mechanical terms in (6). Moreover, taking into account that $\Lambda g_{M N}$ in de Sitter spaces may be viewed as a contribution to the stress tensor $T_{M N}^{\mathrm{vac}}$ of the vacuum, one observes that the decoherence term in (6) may be considered as a quantum version of the (integrated over space time) trace of this tensor, thereby being proportional to the global conformal anomaly of the de Sitter space-time vacuum.

The above results are in full agreement with the violation of CPT in decoherent field theories characterised by an evolution of pure to mixed quantum states, which we have here due to the presence of the Hubble horizon [4]. Indeed, according to a mathematical theorem by R. Wald [12], the CPT operator is not a well-defined quantum mechanical operator in field theories where there is decoherence, that is evolution of pure to mixed states. This leads to a violation of CPT symmetry in its strong form, or rather microscopic time irreversibility. This may lead to different decoherent parameters eventually between particles and antiparticles, reflecting the different ways of interaction with the foam between the two sectors.
We should remark here that, in the case of two-state systems, such as two generation neutrino oscillation models, the double commutator terms proportional to the cosmological constant in (6) may be expressed in terms of metric variations $\left(\Delta g_{M N}\right)^{2}$ between, say, neutrino energy eigenstates, expressing back reaction of neutrino fluctuations onto the space time, as a consequence of interaction with the foam [13]. The induced CPT violation may in general imply, then, metric variations of different strength between particle and antiparticle sectors.

On the other hand, as stated in [12], it could be possible that, despite the strong violation of CPT, a weaker form of CPT invariance is maintained phenomenologically, in the sense that an observer can always prepare pure initial states $|\phi\rangle$, which could evolve to pure final states, $|\psi\rangle$, and for this subset of states the probabilities for the transition and its CPT image were equal:

$$
P(\phi \rightarrow \psi)=P\left(\theta^{-1} \psi \rightarrow \theta \phi\right)
$$

where $\theta$ is the anti-unitary CPT operator acting on pure states only. Such an issue can be disentangled experimentally, and this is the next topic in our discussion.

\section{CHECKING MICROSCOPIC TIME IRREVERSIBILITY IN THE LAB: NEUTRINOS}

The most sensitive, and physically interesting, particle probe for quantum-gravity decoherence, to date, appears to be the neutrino, for which recently there is mounting experimental evidence that it carries a non-trivial mass. The inequality of neutrino masses among the various flavours leads to oscillations, whose properties are affected by the above-mentioned decoherent evolution.

In general, decoherent evolution may be induced by other means, such as the presence of ordinary matter, which the particle passes through, or the presence of quantum space-time foam situation, in which microscopic (Planck size) topologically non-trivial metric fluctuations may make the ground state of quantum gravity behave as a 'medium'. Such contributions are in general independent of the above-described cosmological CPT Violation, although, as we shall discuss below, there might be a common origin of both quantum spacetime foam decoherence and cosmological constant in the following sense: according to some speculative scenaria [14], a neutrino mass difference, and hence flavour mixing, might be the result of quantum gravity decoherence, in analogy with the celebrated MSW effect [15], where contributions to the mass difference between neutrino flavours is induced as a result of the passage of neutrinos through ordinary media. In some approach to the quantisation of flavour mixing in field theory $[16,17]$, as we shall discuss in the next section, one can show that there are non-trivial non-perturbative contributions to the vacuum energy of the Universe (cosmological constant) from massive neutrinos, which are in fact proportional to the (sum) of the mass differences, at least in hierarchical neutrino models [14]. According to our discussion above, then, this would imply cosmological CPT Violation, pointing to the inapplicability of flat-space methods for the quantisation of the 
flavour space, which is thus becoming a curved-space time (de Sitter) problem, awaiting solution.

From a phenomenological viewpoint, one can adopt a model-independent approach to arrive at the master equation for the time evolution of the neutrino density matrix, which could encompass all such foam or cosmological constant CPT Violating effects in a unified formalism, without the necessity for a detailed microscopic knowledge of the underlying physics for the 'environment'. For three generation neutrinos this has been done in [13], and we next proceed to review briefly the situation.

The mathematical formalism adopted is the so-called Lindblad or mathematical semi-groups approach to decoherence [18], which is a very efficient way of studying open systems in quantum mechanics. The time irreversibility in the evolution of such semigroups, which is linked to decoherence, is inherent in the mathematical property of the lack of an inverse in the semigroup. This approach has been followed for the study of quantum-gravity decoherence in the case of neutral kaons in $[19,20]$.

The Lindblad approach to decoherence does not require any detailed knowledge of the environment, apart from energy conservation, entropy increase and complete positivity of the (reduced) density matrix $\rho(t)$ of the subsystem under consideration. The basic evolution equation for the (reduced) density matrix of the subsystem in the Lindblad approach is linear in $\rho(t)$ and reads:

$$
\frac{\partial \rho}{\partial t}=-i\left[H_{\mathrm{eff}}, \rho\right]+\frac{1}{2} \sum_{j}\left(\left[b_{j}, \rho(t) b_{j}^{\dagger}\right]+\left[b_{j} \rho(t), b_{j}^{\dagger}\right]\right),
$$

where $H_{\text {eff }}$ is the effective Hamiltonian of the subsystem, and the operators $b_{j}$ represent the interaction with the environment, and are assumed bounded. Notice that the Lindblad part cannot be written as a commutator (of a Hamiltonian function) with $\rho$. Environmental contributions that can be cast in Hamiltonian evolution (commutator form) are absorbed in $H_{\text {eff }}$.

It must be noted at this stage that the requirement of complete positivity, which essentially pertains to the positivity of the map $\rho(t)$ as the time evolves in the case of many particle situations, such as meson factories (two-kaon states ( $\phi$ factory), or two- $B$-meson states etc.), may not be an exact property of quantum gravity, whose interactions with the environment could be non linear [9]. Nevertheless, complete positivity leads to a convenient and simple parametrization, and it has been assumed so far in many phenomenological analyses of quantum gravity decoherence in generic two state systems, such as two-flavor neutrino systems [21-23].

Formally, the bounded Lindblad operators of an $N$-level quantum mechanical system can be expanded in a basis of matrices satisfying standard commutation relations of Lie groups. For a two-level system [19, 20] such matrices are the SU(2) generators (Pauli matrices) plus the $2 \times 2$ identity operator, while for a three level system [24], which will be relevant for our purposes in this article, the basis comprises of the eight Gell-Mann $S U(3)$ matrices $\Lambda_{i}, i=1, \ldots 8$ plus the $3 \times 3$ identity matrix $I_{3 \times 3}$.

Let $\mathcal{J}_{\mu}, \mu=0, \ldots 8(3)$ be a set of $\mathrm{SU}(3)$ (SU(2)) generators for a three(two)-level system; then, one may expand the vari- ous terms in (8) in terms of $\mathcal{J}_{\mu}$ to arrive at the generic form:

$$
\begin{aligned}
& \frac{\partial \rho_{\mu}}{\partial t}=\sum_{i j} h_{i} \rho_{j} f_{i j \mu}+\sum_{v} \mathcal{L}_{\mu v} \rho_{\mu}, \\
& \mu, \nu=0, \ldots N^{2}-1, \quad i, j=1, \ldots N^{2}-1
\end{aligned}
$$

with $N=3(2)$ for three(two) level systems, and $f_{i j k}$ the structure constants of the $S U(N)$ group. The requirement for entropy increase implies the hermiticity of the Lindblad operators $b_{i}$, as well as the fact that the matrix $\mathcal{L}$ of the the non-Hamiltonian part of the evolution has the properties that $\mathcal{L}_{0 \mu}=\mathcal{L}_{\mu 0}=0, \mathcal{L}_{i j}=\frac{1}{2} \sum_{k, \ell, m} b_{m}^{(n)} b_{k}^{(n)} f_{i m k} f_{\ell k j}$, with the notation $b_{j} \equiv \sum_{\mu} b_{\mu}^{(j)} g_{\mu}$.

In the two-level case of [19] the decoherence matrix $\mathcal{L}_{\mu v}$ is parametrised by a $4 \times 4$ matrix, whose non vanishing entries are occupied by the three parameters with the dimensions of energy $\alpha, \beta, \gamma$ with the properties mentioned above. If the requirement of a completely positive map $\rho(t)$ is imposed, then the $4 \times 4$ matrix $\mathcal{L}$ becomes diagonal, with only one non vanishing entry occupied by the decoherence parameter $\gamma>0$ [23].

In [13] the CPT Violation feature of space-time foam decoherence, has been taken into account for the neutrino oscillation case, by assuming different decoherence parameters between particle and antiparticle sectors. Below we shall use the barred notation for the antiparticle sector quantities. Notice that this is possible in neutrino oscillations because we are dealing with oscillations among flavours separately between particle and antiparticle sectors, e.g. we shall be interested in probabilities $P_{v_{\alpha} \rightarrow v_{\beta}}$, or $P_{\bar{v}_{\alpha} \rightarrow \bar{v}_{\beta}}$, where $\alpha, \beta$ are neutrino flavours. In contrast, in neutral meson systems $[19,20]$, one is dealing with oscillations between particle antiparticle sectors (e.g. $K^{0} \rightarrow \bar{K}^{0}$ for Kaons, etc.), and hence the relevant decoherent evolution contains only one set of parameters $(\alpha, \beta, \gamma)$ for both sectors.

The extension of the completely positive decoherence scenario to the standard three-generation neutrino oscillations case requires formally the adoption of the three-state Lindblad problem. The relativistic neutrino Hamiltonian $H_{\text {eff }} \sim p^{2}+$ $m^{2} / 2 p$, with $m$ the neutrino mass, has been used as the Hamiltonian of the subsystem in the evolution of eq.(8). In terms of the generators $\mathcal{g}_{\mu}, \mu=0, \ldots 8$ of the $\mathrm{SU}(3)$ group, $H_{\mathrm{eff}}$ can be expanded as [24]: $\mathcal{H}_{\mathrm{eff}}=\frac{1}{2 p} \sqrt{2 / 3}\left(6 p^{2}+\sum_{i=1}^{3} m_{i}^{2}\right) \mathcal{I}_{0}+$ $\frac{1}{2 p}\left(\Delta m_{12}^{2}\right) g_{3}+\frac{1}{2 \sqrt{3} p}\left(\Delta m_{13}^{2}+\Delta m_{23}^{2}\right) g_{8}$, with the obvious notation $\Delta m_{i j}^{2}=m_{i}^{2}-m_{j}^{2}, i, j=1,2,3$.

The analysis of [24] assumed ad hoc a diagonal form for the $9 \times 9$ decoherence matrix $\mathcal{L}$ in (9):

$$
\left[\mathcal{L}_{\mu v}\right]=\operatorname{Diag}\left(0,-\gamma_{1},-\gamma_{2},-\gamma_{3},-\gamma_{4},-\gamma_{5},-\gamma_{6},-\gamma_{7},-\gamma_{8}\right)
$$

in direct analogy with the two-level case of complete positivity [21, 23]. As we have mentioned already, there is no strong physical motivation behind such restricted forms of decoherence. This assumption, however, leads to the simplest possible decoherence models, and, for our phenomenological purposes in [13] and here, we will assume the above form and use it to fit all the available neutrino data. It must be clear to 
the reader though, that such a simplification, if proven to be successful (which, as we shall argue below, is the case here), just adds more in favour of decoherence models, given the restricted number of available parameters for the fit in this case. In fact, any other non-minimal scenario will have it easier to accommodate data because it will have more degrees of freedom available for such a purpose.

In this formalism, the neutrino transition probabilities read $[13,24]$ :

$$
\begin{aligned}
& P\left(v_{\alpha} \rightarrow v_{\beta}\right)=\operatorname{Tr}\left[\rho^{\alpha}(t) \rho^{\beta}\right]= \\
& \frac{1}{3}+\frac{1}{2} \sum_{i, k, j} e^{\lambda_{k} t} \mathcal{D}_{i k} \mathcal{D}_{k j}^{-1} \rho_{j}^{\alpha}(0) \rho_{i}^{\beta}
\end{aligned}
$$

where $\alpha, \beta=e, \mu, \tau$ stand for the three neutrino flavors, and Latin indices run over $1, \ldots 8$. The quantities $\lambda_{k}$ are the eigenvalues of the matrix $\mathcal{M}$ appearing in the evolution (9), after taking into account probability conservation, which decouples $\rho_{0}(t)=\sqrt{2 / 3}$, leaving the remaining equations in the form: $\partial \rho_{k} / \partial t=\sum_{j} \mathcal{M}_{k j} \rho_{j}$. The matrices $\mathcal{D}_{i j}$ are the matrices that diagonalise $\mathcal{M}$ [18]. Explicit forms of these matrices, the eigenvalues $\lambda_{k}$, and consequently the transition probabilities (11), are given in [24].

The important point to stress is that, in generic models of oscillation plus decoherence, the eigenvalues $\lambda_{k}$ depend on both the decoherence parameters $\gamma_{i}$ and the mass differences $\Delta m_{i j}^{2}$. For instance, $\lambda_{1}=\frac{1}{2}\left[-\left(\gamma_{1}+\gamma_{2}\right)-\sqrt{\left(\gamma_{2}-\gamma_{1}\right)^{2}-4 \Delta_{12}^{2}}\right]$, with the notation $\Delta_{i j} \equiv \Delta m_{i j}^{2} / 2 p, i, j=1,2,3$. Note that, to leading order in the (small) squared-mass differences, one may replace $p$ by the total neutrino energy $E$, and this will be understood in what follows. We note that $\lambda_{k}$ depend on the quantities $\Omega_{i j}$ :

$$
\begin{aligned}
& \Omega_{12}=\sqrt{\left(\gamma_{2}-\gamma_{1}\right)^{2}-4 \Delta_{12}^{2}} \\
& \Omega_{13}=\sqrt{\left(\gamma_{5}-\gamma_{4}\right)^{2}-4 \Delta_{13}^{2}} \\
& \Omega_{23}=\sqrt{\left(\gamma_{7}-\gamma_{6}\right)^{2}-4 \Delta_{23}^{2}}
\end{aligned}
$$

From the above expressions for the eigenvalues $\lambda_{k}$, it becomes clear that, when decoherence and oscillations are present simultaneously, one should distinguish two cases, according to the relative magnitudes of $\Delta_{i j}$ and $\Delta \gamma_{k l} \equiv \gamma_{k}-\gamma_{l}$ : (i) $2\left|\Delta_{i j}\right| \geq$ $\left|\Delta \gamma_{k \ell}\right|$, and (ii) $2\left|\Delta_{i j}\right|<\left|\Delta \gamma_{k \ell}\right|$. In the former case, the probabilities (11) contain trigonometric (sine and cosine) functions, whilst in the latter they exhibit hyperbolic sin and cosine dependence.

Assuming mixing between the flavors, amounts to expressing neutrino flavor eigenstates $\left|v_{\alpha}\right\rangle, \alpha=e, \mu, \tau$ in terms of mass eigenstates $\left|v_{i}\right\rangle, i=1,2,3$ through a (unitary) matrix $U:\left|v_{\alpha}>=\sum_{i=1}^{3} U_{\alpha i}^{*}\right| v_{i}>$. This implies that the density matrix of a flavor state $\rho^{\alpha}$ can be expressed in terms of mass eigenstates as: $\rho^{\alpha}=\left|v_{\alpha}><v_{\alpha}\right|=\sum_{i, j} U_{\alpha i}^{*} U_{\alpha j}\left|v_{i}><v_{j}\right|$. From this we can determine $\rho_{\mu}^{\alpha}=2 \operatorname{Tr}\left(\rho^{\alpha} \mathcal{g}_{\mu}\right)$, a quantity needed to calculate the transition probabilities (11).

Due to CPT Violation, as mentioned above, we should notice at this stage that, when considering the above probabili- ties in the antineutrino sector, the respective decoherence parameters $\bar{\gamma}_{i}$ in general may be different from the corresponding ones in the neutrino sector, as a result of the strong form of CPT violation. This will be crucial for accommodating [13] the LSND result [25] without conflicting with the rest of the available neutrino data. This feature is totally unrelated to mass differences between flavors.

Compatibility of all available neutrino data, including CHOOZ [26] and LSND [25], can be achieved through a set of decoherence parameters $\gamma_{j}$ in (10) such that: all the $\gamma_{i}$ in the neutrino sector are set to zero, restricting in this way all the decoherence effects to the antineutrino one where:

$$
\begin{aligned}
& \overline{\gamma_{1}}=\overline{\gamma_{2}}=\overline{\gamma_{4}}=\overline{\gamma_{5}} \\
& \quad \text { and } \\
& \overline{\gamma_{3}}=\overline{\gamma_{6}}=\overline{\gamma_{7}}=\overline{\gamma_{8}},
\end{aligned}
$$

For the decoherence parameters we have chosen (c.f. (13))

$$
\overline{\gamma_{1}}=2 \cdot 10^{-18} \cdot E \text { and } \overline{\gamma_{3}}=1 \cdot 10^{-24} / E,
$$

In the above formulae $E$ is the neutrino energy, and barred quantities refer to the antineutrinos. This parametrisation guarantees positivity of the relevant probabilities. Overall, we have introduced only two new parameters, two new degrees of freedom, $\overline{\gamma_{1}}$ and $\overline{\gamma_{3}}$, which, as argued in [13] was sufficient to account for the available experimental data, including the "anomalous" LSND results. Furthermore, we have also set the $\mathrm{CP}$ violating phase of the NMS matrix to zero, so that all the mixing matrix elements become real.

Since the neutrino sector does not suffer from decoherence, there is no need to include the solar data into the fit. We are guaranteed to have an excellent agreement with solar data, as long as we keep the relevant mass difference and mixing angle within the LMA region, something which we shall certainly do.

As mentioned previously, CPT violation is driven by, and restricted to, the decoherence parameters, and hence masses and mixing angles are the same in both sectors, and selected to be

$$
\begin{gathered}
\Delta m_{12}^{2}=\Delta{\overline{m_{12}}}^{2}=7 \cdot 10^{-5} \mathrm{eV}^{2}, \\
\Delta m_{23}^{2}=\Delta{\overline{m_{23}}}^{2}=2.5 \cdot 10^{-3} \mathrm{eV}^{2}, \\
\theta_{23}=\overline{\theta_{23}}=\pi / 4, \theta_{12}=\overline{\theta_{12}}=.45, \\
\theta_{13}=\overline{\theta_{13}}=.05,
\end{gathered}
$$

as indicated by the state of the art analysis.

At this point it is important to stress that the inclusion of two new degrees of freedom is not sufficient to guarantee that one will indeed be able to account for all the experimental observations. We have to keep in mind that, in no-decoherence situations, the addition of a sterile neutrino (which comes along with four new degrees of freedom -excluding again the possibility of CP violating phases) did not seem to be sufficient for matching all the available experimental data, at least in CPT conserving situations.

In order to test our model with these two decoherence parameters in the antineutrino sector, we have calculated the zenith angle dependence of the ratio "observed/(expected in the no oscillation case)", for muon and electron atmospheric neutrinos, for the sub-GeV and multi-GeV energy ranges, when 
mixing is taken into account. Since matter effects are important for atmospheric neutrinos, we have implemented them through a two-shell model, where the density in the mantle (core) is taken to be roughly $3.35(8.44) \mathrm{gr} / \mathrm{cm}^{3}$, and the core radius is taken to be $2887 \mathrm{~km}$. We should note at this stage that a "fake" CPT Violation appears due to matter effects, arising from a relative sign difference of the matter potential between the respective interactions of neutrinos and antineutrinos with ordinary matter. This, however, is easily disentangled from our genuine (due to quantum gravity) CPT Violation, used here to parametrise our model fit to LSND results; indeed, a systematic study of such effects [27] has shown that "fake" CPT Violation increases with the oscillation length, but decreases with the neutrino energy, $E$, vanishing in the limit $E \rightarrow \infty$; moreover, no independent information regarding such effects can be obtained by looking at the antineutrino sector, as compared with data from the neutrino sector, due to the fact that in the presence of "fake" CPT Violation, but in the absence of any genuine CPT breaking, the pertinent CPT probability differences between neutrinos and antineutrinos are related, $\Delta P_{\alpha \beta}^{\mathrm{CPT}}=-\Delta P_{\bar{\beta} \bar{\alpha}}^{\mathrm{CPT}}$, where $\Delta P_{\alpha \beta}^{\mathrm{CPT}}=P_{\alpha \beta}-P_{\bar{\beta} \bar{\alpha}}$, and the Greek indices denote neutrino flavors. These features are to be contrasted with our dominant decoherence effects $\bar{\gamma}_{1}$ (14), proportional to the antineutrino energy, $E$, which are dominant only in the antineutrino sector. For the same reason, our effects can be disentangled from "fake" decoherence effects arising from Gaussian averages of the oscillation probability due to, say, uncertainties in the energy of the neutrino beams [28], which are the same for both neutrinos and antineutrinos. We, therefore, claim that the complex energy dependence in (14), with both $L \cdot E$ and $L / E$ terms being present in the antineutrino sector, may be a characteristic feature of new physics, with the $L \cdot E$ terms being related to quantumgravity induced (genuine) CPT Violating decoherence.

The results are shown in Fig. 1 (c), where, for the sake of comparison, we have also included the experimental data. We also present in that figure the pure decoherence scenario in the antineutrino sector (a), as well as in both sectors (b). For completeness, we also present a scenario with neutrino mixing but with decoherence operative in both sectors (d). The conclusion is straightforward: pure decoherence is wildly excluded, while decoherence plus mixing provides an astonishing agreement with experiment.

As bare eye comparisons can be misleading, we have also calculated the $\chi^{2}$ value for each of the cases [13].From this analysis it becomes clear that the mixing plus decoherence scenario in the antineutrino sector can easily account for all the available experimental information, including LSND. It is important to stress once more that our sample point was not obtained through a scan over all the parameter space, but by an educated guess, and therefore plenty of room is left for improvements. On the other hand, for the mixing-only/nodecoherence scenario, we have taken the best fit values of the state of the art analysis and therefore no significant improvements are expected. At this point a word of warning is in order: although superficially it seems that scenario (d), decoherence plus mixing in both sectors, provides an equally good fit, one should remember that including decoherence effects in the neutrino sector can have undesirable effects in solar neutrinos, especially due to the fact that decoherence effects are weighted by the distance travelled by the neutrino, something that may lead to seisable (not observed!) effects in the solar case.

One might wonder then, whether decohering effects, which affect the antineutrino sector sufficiently to account for the LSND result, have any impact on the solar-neutrino related parameters, measured through antineutrinos in the KamLAND experiment [29]. In order to answer this question, it will be sufficient to calculate the electron survival probability for KamLAND in our model, which turns out to be $\left.P_{\bar{v}_{\alpha} \rightarrow \bar{v}_{\beta}}\right|_{\text {KamLAND }} \simeq .63$, in perfect agreement with observations. As is well known, KamLAND is sensitive to a bunch of different reactors with distances spanning from 80 to $800 \mathrm{~km}$. However, the bulk of the signal comes from just two of those, whose distances are 160 and $179 \mathrm{~km}$. These parameters have been used to compute the survival probability. It is also interesting to notice that in our model, the LSND effect is not given by the phase inside the oscillation term ( which is proportional to the solar mass difference) but rather by the decoherence factor multiplying the oscillation term. Therefore the tension between LSND and KARMEN [30] data is naturally eliminated, because the difference in length leads to an exponential suppression. Another potential source of concern for the present model of decoherence might be accelerator neutrino experiments, which involve high energies and long baselines, and where the decoherence $L \cdot E$ scaling can potentially be probed. This, however, is not the case. Accelerator experiments typically join their neutrino and antineutrino data, with the antineutrino statistics being always smaller than the neutrino one. This fact, together with the smaller antineutrino cross section, renders our potential signal consistent with the background contamination. Even more, in order to constrain decoherence effects of the kind we are proposing here through accelerator experiments, excellent control and knowledge of the beam background are mandatory. The new KTeV data [31] on kaon decay branching ratios, for example, will change the $v_{e}$ background enough to make any conclusion on the viability of decoherence models useless. After all, the predicted signal in our decoherence scenario will be at the level of the electron neutrino contamination, and therefore one would need to disentangle one from the other.

Having said that, it is now clear that decoherence models (once neutrino mixing is taken into account) are the best (and arguably the only) way to explain all the observations including the LSND result. This scenario, which makes dramatic predictions for the upcoming neutrino experiments, expresses a strong observable form of CPT violation in the laboratory, and in this sense, our fit gives a clear answer to the question asked in the introduction as to whether the weak form of CPT invariance (7) is violated in Nature. It seems that, in order to account for the LSND results, we should invoke such a decoherence-induced CPT violation, which however is independent of any mass differences between particles and antiparticles.

This CPT violating pattern, with equal mass spectra for neutrinos and antineutrinos, will have dramatic signatures in 

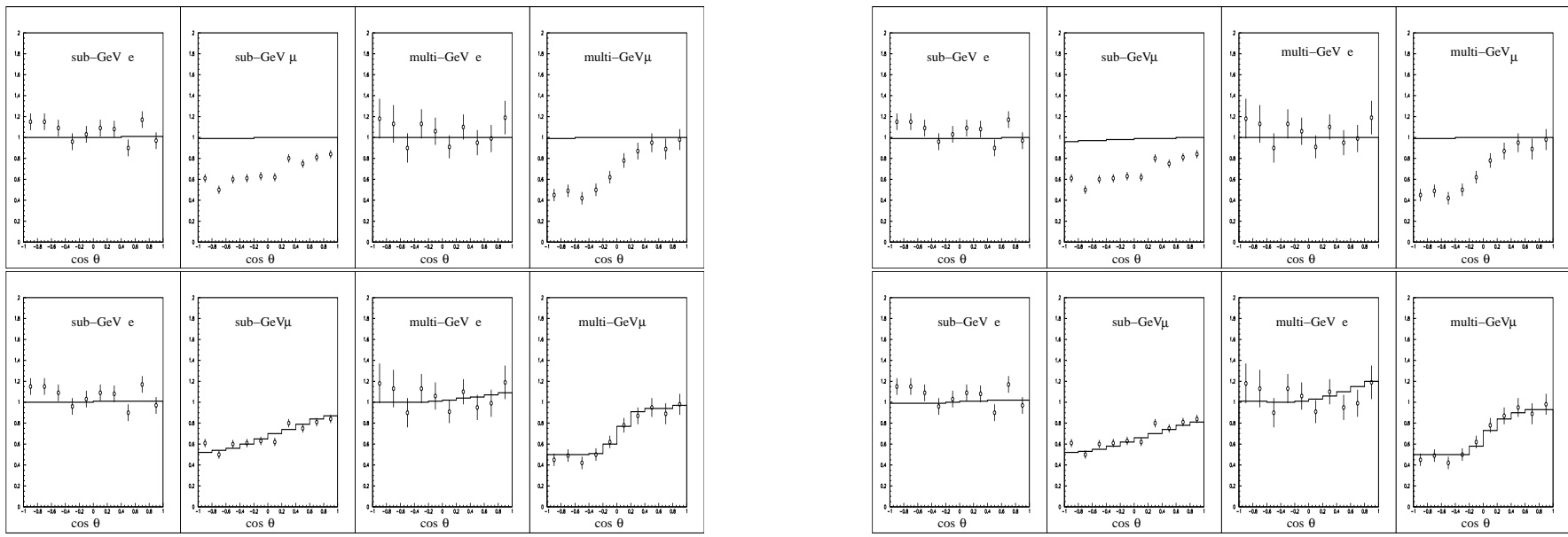

FIG. 1: Decoherence fits, from top left to bottom right: (a) pure decoherence in antineutrino sector, (b) pure decoherence in both sectors, (c) mixing plus decoherence in the antineutrino sector only, (d) mixing plus decoherence in both sectors. The dots correspond to SK data.

future neutrino oscillation experiments. The most striking consequence will be seen in MiniBooNE [32], According to our picture, MiniBooNE will be able to confirm LSND only when running in the antineutrino mode and not in the neutrino one, as decoherence effects live only in the former. Smaller but experimentally accessible signatures will be seen also in MINOS [33], by comparing conjugated channels (most noticeably, the muon survival probability). We should mention at this stage that our model is in agreement with the strong suppression of decoherence in the neutrino sector expected from astrophysical observations of high energy cosmic neutrinos [34].

We next remark that fits with decoherence parameters with energy dependences of the form (14) imply that the exponential factors $e^{\lambda_{k} t}$ in (11) due to decoherence will modify the amplitudes of the oscillatory terms due to mass differences, and while one term depends on $L / E$ the other one is driven by $L \cdot E$, where we have set $t=L$, with $L$ the oscillation length (we are working with natural units where $c=1$ ).

The order of the coefficients of these quantities, $\gamma_{j}^{0} \sim$ $10^{-18}, 10^{-24}(\mathrm{GeV})^{2}$, found in our sample point, implies that for energies of a few $\mathrm{GeV}$, which are typical of the pertinent experiments, such values are not far from $\gamma_{j}^{0} \sim \Delta m_{i j}^{2}$. If our conclusions survive the next round of experiments, and therefore if MiniBOONE experiment [32] confirms previous LSND claims, then this may be a significant result. One would be tempted to conclude that if the above estimate holds, this would probably mean that the neutrino mass differences might be due to quantum gravity decoherence. Theoretically it is still unknown how the neutrinos acquire a mass, or what kind of mass (Majorana or Dirac) they possess. There are scenaria in which the mass of neutrino may be due to some peculiar backgrounds of string theory for instance. If the above model turns out to be right we might then have, for the first time in low energy physics, an indication of a direct detection of a quantum gravity effect, which disguised itself as an induced decohering neutrino mass difference. Notice that in our sample point only antineutrinos have non-trivial decoherence parameters $\overline{\gamma_{i}}$, for $i=1$ and 3 , while the corresponding quantities in the neutrino sector vanish. This implies that there is a single cause for mass differences, the decoherence in antineutrino sector, which is compatible with common mass differences in both sectors. If this turns out to be true, it could then lead to important conceptual changes in our thinking of the problem of particle masses in field theory.

\section{NEUTRINO MIXING, SPACE-TIME FOAM AND COSMOLOGICAL CONSTANT?}

In what follows we will make this assumption, namely that decoherence effects, due to interactions with the foam, contribute to the Hamiltonian terms in the evolution of the neutrino density matrix, and result in neutrino mass differences in much the same way as the celebrated MSW effect[15], responsible for a neutrino mass splitting due to interactions with a medium. Indeed, when neutrinos travel through matter, the neutral current contribution to this interaction, proportional to $-G_{F} n_{n} / \sqrt{2}$, with $G_{F}$ Fermi's weak interaction constant, and $n_{n}$ the neutron density in the medium, is present for both $v_{e}$ and $v_{\mu}$ (in a two flavour scenario), while the charged current contribution, given by $\sqrt{2} G_{F} n_{e}$, with $n_{e}$ the medium's electronic density, is present only for $v_{e}$. The flavour eigenstates $v_{e, \mu}$ can then be expressed in terms of fields $\tilde{v}_{1,2}$ with definite masses $\tilde{m}_{1,2}$ respectively, with a mixing angle $\tilde{\theta}$, the tilde notation indicating the effects of matter. The tilded quantities are diagonalised with respect to the Hamiltonian of $\mathrm{v}_{e}, \mathrm{v}_{\mu}$ in the presence of non-trivial matter media, and one can find the following relations between vacuum (untilded) and medium parameters[15] $\sin ^{2} 2 \tilde{\theta} \simeq \sin ^{2} 2 \theta\left(\frac{\Delta m^{2}}{\Delta \tilde{m}^{2}}\right)$, with $\Delta \tilde{m}^{2}=\sqrt{\left(D-\Delta m^{2} \cos 2 \theta\right)^{2}+\left(\Delta m^{2} \sin 2 \theta\right)^{2}}, D=2 \sqrt{2} G_{F} n_{e} k$. From this we observe that the medium-induced effects in the mass splittings are proportional to the electronic density of the medium and in fact, even if the neutrinos would have been mass degenerate in vacuum, such a degeneracy would be lifted 
by a medium.

To get a qualitative idea of what might happen with the foam, one imagines a similar mixing for neutrinos, as a result of their interaction with a quantum-gravity decohering foam situation. As a result, there are gravitationally-induced effective masses for neutrinos, due to flavour dependent interactions of the foam, which are in principle allowed in quantum gravity. In analogy (but we stress that this is only an analogy) with the MSW effect, the gravitationally-induced mass-splitting effects are expected now to be proportional to $G_{N} n_{\mathrm{bh}} k$, where $G_{N}=1 / M_{P}^{2}$ is Newton's constant, $M_{P} \sim 10^{19}$ $\mathrm{GeV}$ is the quantum gravity scale, and $n_{b h}$ is a "foam" density of appropriate space time defects (such as Planck size black holes etc.), whose interaction with the neutrinos discriminates between flavours, in an analogous way to the matter effect. Neutrinos, being electrically neutral can indeed interact nontrivially with a space time foam, and change flavour as a result of such interactions, since such processes are allowed by quantum gravity. On the other hand, due to electric charge conservation of microscopic black holes, quarks and charged leptons, cannot interact non-trivially with the foam. In this spirit, one can imagine a microscopic charged black-hole/antiblack-hole pair being created by the foam vacuum. Evaporation of these black holes (probably at a slower rate than their neutral counterparts, due to their near extremal nature [35]) can produce preferentially $e^{+} e^{-}$pairs (lighter than muons), of which the positrons, say, are absorbed into the microscopic event horizons of the evaporating charged anti-black hole. This leaves us with a stochastically fluctuating (about a mean value) electron (or more general charge) density, $n_{\mathrm{bh}}^{c}(r)$, induced by the gravitational foam, $\left\langle n_{\mathrm{bh}}^{c}(r)\right\rangle=n_{0} \neq 0$, $\left\langle n_{\mathrm{bh}}^{c}(r) n_{\mathrm{bh}}^{c}\left(r^{\prime}\right)\right\rangle \neq 0$, which, in analogy with the electrons of the MSW effect in a stochastically fluctuating medium[36], can interact non-trivially only with $v_{e}$ but not with the $v_{\mu}$, in contrast to neutral black holes which can interact with all types of neutrinos[14]. We assume, of course, that the contributions to the vacuum energy that may result from such emission and absorption processes by the black holes in the foamy vacuum are well within the known limits. For instance, one may envisage supersymmetric/superstring models of space-time foam, where such contributions may be vanishingly small[37]. The mean value (macroscopic) part, $n_{0}$, of $n_{\mathrm{bh}}^{c}(r)$, assumed time independent, will contribute to the Hamiltonian part of the evolution of the neutrino density matrix, $\rho$. In analogy with the (stochastic) MSW effect[15, 36], this part yields space-time foam-induced mass-squared splittings for neutrinos:

$$
\left\langle\Delta m_{\text {foam }}^{2}\right\rangle \propto G_{N}\left\langle n_{\mathrm{bh}}^{c}(r)\right\rangle k
$$

with non trivial quantum fluctuations ( $k$ is the neutrino momentum scale). To ensure a constant neutrino mass one may consider the case where $\left\langle n_{\mathrm{bh}}^{c}(r)\right\rangle$, which expresses the average number of virtual particles emitted from the foam with which the neutrino interacts, is inversely proportional to the (neutrino) momentum. This is reasonable, since the faster the neutrino, the less the available time to interact with the foam, and hence the smaller the number of foam particles it interacts with. Such flavour-violating foam effects would also contribute to decoherence through the quantum fluctuations of the foam-medium density[14, 36], by means of induced non-Hamiltonian terms in the density-matrix evolution. Such effects assume a double commutator structure[14, 36, 38] and are due to both, the fluctuating parts of the foam density, as well as the effects of the mixing (15) on the vacuum energy. Indeed, as we discussed in [14], and shall review briefly below, neutrino flavour mixing may lead to a non-trivial contribution to the vacuum energy, in a non-perturbative way suggested in [17]. Hence, such effects are necessarily CPT violating[11], in the sense of entailing an evolution of an initially pure neutrino quantum state to a mixed one due to the presence of the Hubble horizon associated with the non zero cosmological constant, which prevents pure asymptotic states from being well defined. In that case, CPT is violated in its strong form, that is CPT is not a well-defined operator, according to the theorem of [12].

For convenience we shall discuss explicitly the twogeneration case. The arguments can be extended to three generations, at the expense of an increase in mathematical complexity, but will not affect qualitatively the conclusions drawn from the two-generation case. The arguments are based on the observation[16] that in quantum field theory, which by definition requires an infinite volume limit, in contrast to quantum mechanical treatment of fixed volume[39], the neutrino flavour states are orthogonal to the energy eigenstates, and moreover they define two inequivalent vacua related to each other by a non unitary transformation $G^{-1}(\theta, t)$ : $|0(t)\rangle_{f}=G_{\theta}^{-1}(t)|0(t)\rangle_{m}$, where $\theta$ is the mixing angle, $t$ is the time, and the suffix $\mathrm{f}(\mathrm{m})$ denotes flavour(energy) eigenstates respectively, and $G_{\theta}^{-1}(t) \neq G_{\theta}^{\dagger}(t)$ is a non-unitary operator expressed in terms of energy-eigenstate neutrino free fields $v_{1,2}$ [17]: $G_{\theta}(t)=\exp \left(\theta \int d^{3} x\left[v_{1}^{\dagger}(x) v_{2}(x)-v_{2}^{\dagger}(x) v_{1}(x)\right]\right)$. A rigorous mathematical analysis of this problem has also appeared in [40]. As a result of the non unitarity of $G_{\theta}^{-1}(t)$, there is a Bogolubov transformation[16] connecting the creation and annihilation operator coefficients appearing in the expansion of the appropriate neutrino fields of the energy or flavour eigenstates. Of the two Bogolubov coefficients appearing in the treatment, we shall concentrate on $V_{\vec{k}}=\left|V_{\vec{k}}\right| e^{i\left(\omega_{k, 1}+\omega_{k, 2}\right) t}$, with $\omega_{k, i}=\sqrt{k^{2}+m_{i}^{2}}$, the (positive) energy of the neutrino energy eigenstate $i=1,2$ with mass $m_{i}$. This function is related to the condensate content of the flavour vacuum, in the sense of appearing in the expression of an appropriate non-zero number operator of the flavour vacuum[17, 40]: ${ }_{f}\left\langle 0\left|\alpha_{\vec{k}, i}^{r \dagger} \alpha_{\vec{k}, i}^{r}\right| 0\right\rangle_{f}={ }_{f}\left\langle 0\left|\beta_{\vec{k}, i}^{r \dagger} \beta_{\vec{k}, i}^{r}\right| 0\right\rangle_{f}=\sin ^{2} \theta\left|V_{\vec{k}}\right|^{2}$ in the twogeneration scenario [16]. $\left|V_{\vec{k}}\right|$ has the property of vanishing for $m_{1}=m_{2}$, it has a maximum at the momentum scale $k^{2}=m_{1} m_{2}$, and for $k \gg \sqrt{m_{1} m_{2}}$ it goes to zero as:

$$
\left|V_{\vec{k}}\right|^{2} \sim \frac{\left(m_{1}-m_{2}\right)^{2}}{4|\vec{k}|^{2}}, \quad k \equiv|\vec{k}| \gg \sqrt{m_{1} m_{2}}
$$

The analysis of [17] argued that the flavour vacuum $|0\rangle$, is the correct one to be used in the calculation of the average vacuum energy, since otherwise the probability is not conserved[41]. The energy-momentum tensor of a Dirac fermion field in the 
Robertson-Walker space-time background can be calculated straightforwardly in this formalism. The flavour-vacuum average value of its temporal $T_{00}$ component, which yields the required contribution to the vacuum energy due to neutrino mixing, is[17]:

$$
\begin{aligned}
& f\left\langle 0\left|T_{00}\right| 0\right\rangle_{f}=\left\langle\rho_{\mathrm{vac}}^{\nu-\operatorname{mix}}\right\rangle \eta_{00} \\
& =\sum_{i, r} \int d^{3} k \omega_{k, i}\left({ }_{f}\left\langle 0\left|\alpha_{\vec{k}, i}^{r \dagger} \alpha_{\vec{k}, i}^{r}\right| 0\right\rangle_{f}+{ }_{f}\left\langle 0\left|\beta_{\vec{k}, i}^{r \dagger} \beta_{\vec{k}, i}^{r}\right| 0\right\rangle_{f}\right)= \\
& 8 \sin ^{2} \theta \int_{0}^{K} d^{3} k\left(\omega_{k, 1}+\omega_{k, 2}\right)\left|V_{\vec{k}}\right|^{2} .
\end{aligned}
$$

where $\eta_{00}=1$ in a Robertson-Walker (cosmological) metric background. The momentum integral in (17) is cut-off from above at a certain scale, $K$ relevant to the physics of neutrino mixing. In conventional approaches, where the mass generation of neutrino occurs at the electroweak phase transition, this cutoff scale can be put on the electroweak scale $K \sim 100$ $\mathrm{GeV}$, but this yields unacceptably large contributions to the vacuum energy. An alternative scale has been suggested in [17], namely $K \sim \sqrt{m_{1} m_{2}}$ as the characteristic scale for the mixing. In this way these authors obtained a phenomenologically acceptable value for $\left\langle\rho_{\mathrm{vac}}^{v-\text { mix }}\right\rangle$.

In our case we shall use a different cutoff scale [14], which allows for some analytic estimates of (17) to be derived, as being mathematically consistent with the asymptotic form of (16), which is valid in a regime of momenta $k \gg \sqrt{m_{1} m_{2}}$. This cutoff scale is simply given by the sum of the two neutrino masses, $K \equiv k_{0}=m_{1}+m_{2}$, is compatible with our decoherence-induced mass difference scenario, and also allows for a mathematically consistent analytic estimate of the neutrino-mixing contribution to the vacuum energy in this framework. For hierarchical neutrino models, for which $m_{1} \gg m_{2}$, we have that $k_{0} \gg \sqrt{m_{1} m_{2}}$, and thus, if we assume that the modes near the cutoff contribute most to the vacuum energy (17), which is clearly supported by the otherwise divergent nature of the momentum integration, and take into account the asymptotic properties of the function $V_{\vec{k}}$, which are safely valid in this case, we obtain:

$$
\begin{aligned}
& \left\langle\rho_{\mathrm{vac}}^{\nu-\mathrm{mix}}\right\rangle \sim 8 \pi \sin ^{2} \theta\left(m_{1}-m_{2}\right)^{2}\left(m_{1}+m_{2}\right)^{2} \times \\
& \left(\sqrt{2}+1+O\left(\frac{m_{2}^{2}}{m_{1}^{2}}\right)\right) \propto \sin ^{2} \theta\left(\Delta m^{2}\right)^{2}
\end{aligned}
$$

in the limit $m_{2} \ll m_{1}$. For the $(1,2)$ sector, the corresponding $\Delta m^{2}$ is given by the solar neutrino data and is estimated to be $\Delta m_{12}^{2} \simeq 10^{-5} \mathrm{eV}^{2}$, resulting in a contribution of the right order. In this way the cosmological constant $\Lambda$ is elegantly expressed in terms of the smallest (infrared, $\Delta m^{2}$ ) and the largest (ultraviolet, $M_{P}^{2}$ ) Lorentz-invariant mass scales available. It can be argued [14] that the above choice of the cutoff $k_{0} \sim m_{1}+m_{2}$ is consistent with our conjecture on the decoherence origin of the neutrino mass difference, due to interaction with the foam medium (15). Notice that the above way of deriving the neutrino-mixing contribution to the dark energy is independent of the usual perturbative loop arguments, and, in this sense, the result (18) should be considered as exact (non perturbative), if true.
Some important remarks are now in order. First of all, our choice of cutoff scale was such that the resulting contribution to the cosmological constant depends on the neutrino masssquared differences and not on the absolute mass, and hence it is independent of any zero-point energy, in agreement with energy-driven decoherence models [38]. For us, it is curved space physics that is responsible for lifting the mass degeneracy of neutrino mass eigenstates and create the "flavour" problem. This is an important point, which may serve as motivation (not proof) behind such a cutoff "choice", which we conjecture is a physical "necessity". We have argued above that such a cutoff "choice" is a natural one from the point of view of quantum-gravity decoherence-induced mass differences. Detailed models of this fall way beyond the purposes of this brief note. Nevertheless, we believe that the abovedemonstrated self-consistency of this cutoff choice within the remit of our toy model of space time foam is intellectually challenging and encouraging for further studies of this important issue.

The above considerations above were based on the suggestion of ref. [16] on a Fock-like quantisation of the flavour space. There is still controversy in the literature regarding the physical meaning of such quantum flavour states [42], in particular it has been argued that, although such states are mathematically elegant and correct constructions, nevertheless they lead to no observable consequences. However, in view of the results of [17] and of the present work, such an argument may not be correct, since the mass-squared difference contribution to the cosmological constant is an observable (global) consequence of the Fock-like flavour space quantisation. The presence of a time independent cosmological constant (18) in the flavour vacuum, which notably is not present if one uses instead the mass eigenstate vacuum, implies an asymptotic future event horizon for the emerging de-Sitter Universe. The flat-space time arguments of [42] for the flavour space field theory cannot then be applied, at least naively, and the problem of quantisation of the Fock-like flavour space is equivalent to the (still elusive) quantisation of field theories in (curved) de-Sitter space times. In such a case one cannot define properly asymptotic states, and hence a scattering matrix. This will lead to decoherence, in the sense of a modified temporal evolution for matter states.

We now remark that in the case of (anti)neutrinos passing through stochastic media [36], including space time foam [14], there are additional contributions to decoherence, besides the presence of a $\Lambda$-term, which may offer a natural explanation of the decoherence parameters of [13]. An important source of decoherence in such media is due to the uncertainties in the energy $E$ and/or the oscillation length $L$ of the (anti)neutrino beam. In fact, it can be shown [28] that if one averages the standard oscillation probabilities $P_{v_{\alpha} \rightarrow v_{\beta}}$ over Gaussian distributions for $E$ and/or $L$ with a variance $\sigma^{2}$, the result is equivalent to neutrino decoherence models, in the sense of the time dependent profile of the associated probability being identical to that of a completely-positive decoher- 
ence model. One finds for $n$ flavours [27],

$$
\begin{aligned}
& \left\langle P_{\alpha \beta}\right\rangle=\delta_{\alpha \beta}-2 \sum_{a=1}^{n} \sum_{\beta=1, a<b}^{n} \operatorname{Re}\left(U_{\alpha a}^{*} U_{\beta a} U_{\alpha b} U_{\beta b}^{*}\right) \times \\
& \left(1-\cos \left(2 \ell \Delta m_{a b}^{2}\right) e^{-2 \sigma^{2}\left(\Delta m_{a b}^{2}\right)^{2}}\right)- \\
& 2 \sum_{a=1}^{n} \sum_{b=1, a<b}^{n} \operatorname{Im}\left(U_{\alpha a}^{*} U_{\beta a} U_{\alpha b} U_{\beta b}^{*}\right) \times \\
& \sin \left(2 \ell \Delta m_{a b}^{2}\right) e^{-2 \sigma^{2}\left(\Delta m_{a b}^{2}\right)^{2}}
\end{aligned}
$$

where $U$ is the mixing matrix $\ell \equiv\langle x\rangle, \sigma=\sqrt{\left\langle(x-\langle x\rangle)^{2}\right.} \equiv$ $(L / 4 E) r$, and $x=L / 4 E$. The resulting form is identical to that of decoherence, as becomes evident by noting that the exponential damping factors can be written in the form $e^{-\gamma_{j} L}$ with $t=L(c=1)$, and decoherence parameters $\gamma_{j}$ of order: $2 \sigma_{j}^{2}\left(\Delta m^{2}\right)^{2}=\gamma_{j} L$, from which $\gamma_{j}=\frac{\left(\Delta m^{2}\right)^{2}}{8 E^{2}} L r_{j}^{2}$. There are various scenaria that restrict the order of $\sigma$. In general, the acceptable bounds on $\sigma$ may be divided in two major categories, depending on the form of the uncertainties [28]: $\sigma_{j} \simeq \Delta x \simeq$ $\Delta_{j} \frac{L}{4 E} \leq \frac{\langle L\rangle}{4\langle E\rangle}\left(\frac{\Delta_{j} L}{\langle L\rangle}+\frac{\Delta_{j} E}{\langle E\rangle}\right)$, or $\sigma_{j} \leq \frac{\langle L\rangle}{4\langle E\rangle}\left(\left[\frac{\Delta_{j} L}{\langle L\rangle}\right]^{2}+\left[\frac{\Delta_{j} E}{\langle E\rangle}\right]^{2}\right)^{1 / 2}$. In three generation models the values of the length and energy uncertainties may vary between flavours, and also between neutrinos and antineutrinos, as a result of the intrinsic CPT violation, hence the subscript $j$ in the above formulae (for antiparticle sectors it is understood that $j \rightarrow \bar{j}$ ). From the above considerations it becomes clear that, for $L \sim 2 E / \Delta m^{2}$, which is characteristic for oscillations, one has decoherence parameters $\gamma_{j} \sim\left(\Delta m^{2} / E\right) r_{j}^{2}$. It is interesting to estimate first the order of decoherence induced by conventional physics, for instance decoherence induced by uncertainties in the measured energy of the beam due to experimental limitations. For long base line, atmospheric or cosmic neutrino experiments, where $\Delta L / L$ is negligible, and $\Delta E / E \sim 1$ such decoherence parameters are found at most of order $\gamma \sim 10^{-24} \mathrm{GeV}$, for the relevant range of energies, and they diminish with energy, vanishing formally when $E \rightarrow \infty$, which seems to be a general feature of conventional matter-induced decoherence effects [27].

To obtain the decoherence parameters of the best-fit model of [13] it suffices to choose for the antineutrino sector $r_{\overline{3}}=$ $r_{\overline{8}} \sim \Delta E / E \sim 1$, and $r_{\overline{1}}^{2}=r_{\frac{2}{2}}^{2} \sim 10^{-18} \cdot E^{2} / \Delta m^{2}$. As seen above, the decoherence parameters exhibiting a $1 / E$ energy dependence could be attributed to conventional energy uncertainties occurring in the beam of the (anti)neutrinos. However, the parameters proportional to $E$, if true, may be attributed to exotic physics. The fact that $r_{j}$ in general receives contributions from both length and energy uncertainties provides a natural explanation for the different energy dependence of the decoherence parameter of the model of I in the antiparticle sector. Indeed, having identified $r_{\overline{3}}=r_{\overline{8}}$ as decoherence induced by 'conventional-looking' energy uncertainties in the antineutrino sector, it is natural to assume that the $\gamma_{3}=\gamma_{8} \propto E$ decoherence is due to genuine quantum gravity effects, increasing with energy, which are associated with metric tensor quantum fluctuations. This is achieved provided we assume that $r_{\overline{3}}^{2}=r_{\overline{8}}^{2} \sim(\Delta L / L)^{2}$, i.e. these decoherence coefficients are predominantly oscillation-length-uncertainty driven, and take into account that variations in the invariant length may be caused by metric fluctuations, since $L^{2}=g_{\mu v} L^{\mu} L^{\nu}$, implying $\left(\Delta g_{\mu \nu}\right)^{2} \sim(\Delta L)^{2} / L^{2}$, in order of magnitude. To obtain the best fit results of I, then, for $L \sim 2 E / \Delta m^{2}$, one needs quantumgravity induced metric fluctuations in the antineutrino sector of order $\left(\bar{\Delta} g_{\mu \nu}\right)^{2} \sim 10^{-18} L \cdot E$. The increase with energy is not unreasonable, given that the higher the energy of the antineutrino the stronger the back reaction onto space time, and hence the stronger the quantum-gravity induced metric fluctuations. The factor $10^{-18}$ may be thought of as being of order $E / M_{P}$, with $M_{P} \sim 10^{19} \mathrm{GeV}$ the Planck mass, although alternative interpretations may be valid (see discussion on possible cosmological interpretations at the end of the article). The increase with $L$ is not uncommon in stochastic models of quantum foam, where the decoherence 'medium' effects build up with the distance the (anti)particle travels [37]. We also mention at this stage that, apart from these effects, in stochastic models of foam there are additional contributions to decoherence, arising from the fluctuations of the density of the medium. These too can mimic the effects of the best-fit model of [13] in the antineutrino sector, as discussed in some detail in [14], but their $L$-dependence is different from that of the above effects. Comparison between short and long baseline experiments, therefore, may differentiate between the various decoherent contributions.

At this stage we would like to mention that the abovedescribed model of decoherence provides a novel and extremely economical mechanism to generate the observed baryon asymmetry in the Universe [14], through a process of equilibrium electroweak leptogenesis. To this end we first recall that, in the analysis of ref. [20], dealing with decoherent evolution in the neutral Kaon case, the asymmetries between the semileptonic decays of $K_{0}$ and those of $\bar{K}_{0}$ turned out to depend linearly on dimensionless decoherence parameters such as $\hat{\gamma}=\gamma / \Delta \Gamma$; in the parametrisation of Ellis et al. in [19], where $\Delta \Gamma=\Gamma_{L}-\Gamma_{S}$ was a characteristic energy scale associated with energy eigenstates of the kaon system. In fact, the dependence was such that the decoherence corrections to the asymmetry were of order $\widehat{\gamma}$ in complete positivity scenaria, where only one decoherence parameter, $\gamma>0$ was non zero. In similar spirit, in our case of lepton-antilepton number asymmetries, one expects the corresponding asymmetry to depend, to leading order, linearly on the quantity $\widehat{\gamma}=\gamma / \sqrt{\Delta m^{2}}$, since the quantity $\sqrt{\Delta m^{2}}$ is the characteristic energy scale in the neutrino case, playing a role analogous to $\Delta \Gamma$ in the kaon case. The only difference from the kaon case, is that here, in contrast to the kaon asymmetry results, there are no zeroth order terms, and thus the result of the matter-antimatter asymmetry is proportional to the dimensionless decoherence parameter $\widehat{\gamma}$, which we are going to take as the larger of the two dechorence parameters of our model in [13], discussed in the previous section, namely $\widehat{\gamma} \rightarrow \widehat{\gamma_{1}}=10^{-18} \cdot E / \sqrt{\Delta m^{2}}$. In this way, the matter-antimatter asymmetry in the Universe is estimated to be $\mathcal{A}=\frac{\langle v\rangle-\langle\bar{v}\rangle}{\langle v\rangle+\langle\bar{v}\rangle} \simeq \widehat{\gamma_{1}} \simeq 10^{-6}$. The numerical coefficient $10^{-18}$ on $\gamma$ may be thought of as the ratio $T / M_{P}$ with $T$ the temperature, whose value gets frozen at the EW symmetry breaking temperature. Thus, $B=\frac{n_{v}-\overline{n_{v}}}{s} \sim \frac{\mathcal{A} n_{v}}{g_{*} n_{\gamma}}$ with $n_{v}\left(\overline{n_{v}}\right)$ 
the number density of (anti) neutrinos, $n_{\gamma}$ the number density of photons and $g_{*}$ the effective number of degrees of freedom (at the temperature where the asymmetry is developed) which depends on the exact matter content of the model but it ranges from $10^{2}$ to $10^{3}$ in our case. This implies a residual baryon asymmetry of order $10^{-10}$, roughly the desired magnitude.

Finally, before closing we would like to alert the interested reader in another possible aspect of the Fock space quantisation of the flavour vacuum [17], advocated in [43]. In the case of non-trivial neutrino mixing, the flavour Fock states do not satisfy the standard Linear Lorentz invariant dispersion relations $E^{2}=p^{2}+m^{2}$, where $p$ is the momentum and $m$ a rest mass, since they are superpositions of mass eigenstates satisfying standard dispersion relations but with different masses. The idea of [43] is that such states may experience non-linear modifications of the Lorentz symmetry, of which doubly special relativities is one example [44], which should guarantee the frame independence of the results. The remark we would like to make in connection with this, is that, in view of the contributions of the Flavour Fock states to the cosmological constant (18), it may be possible to specify the non-linear modifications of the Lorentz symmetry satisfied by the Fock states, which was not possible for a general mixing angle in [43], by adopting the idea of [45]. According to that, the low-energy limit of a quantum theory of gravity in a space time with a (positive) cosmological constant $\Lambda$, must be a theory which is invariant under a deformed Poincaré symmetry, with the pertinent (dimensionful) deformation parameter being related to the cosmological constant. These arguments are valid as long as the theory behaves smoothly in the limit when the cosmological constant becomes small as compared to the Planck scale (or, in general the scale characteristic of the quantum gravity), i.e. in the limit $\Lambda \ell_{\text {Planck }}^{2} \rightarrow 0$, which is certainly the case of (18). In our case, where the neutrino mass differences have been conjectured to be the result of a space-time foamy situation, such considerations become of great importance in determining the symmetry structure underlying the non-flat space-time quantum field (or string) theory at hand. We hope to study such important issues in detail in a future publication.

\section{ACKNOWLEDGEMENTS}

It is a pleasure to thank the organisers of the DICE2004 conference for the invitation, their support, and for creating such a thought stimulating and pleasant atmosphere during the conference. It is also my pleasure to acknowledge discussions with M. Blasone, A. Capolupo, G. Lambiase, F. Lombardo, R. Rivers, R. Sorkin and G. Vitiello.
[1] B. P. Schmidt et al., Astrophys. J. 507, (1998) 46 [arXiv:astroph/9805200]; S. Perlmutter et al. [Supernova Cosmology Project Collaboration], Astrophys. J. 517, (1999) 565. [arXiv:astro-ph/9812133].

[2] D. N. Spergel et al., Astrophys. J. Suppl. 148, (2003) 175. [arXiv:astro-ph/0302209].

[3] A. A. Starobinsky, Phys. Lett. B 117 (1982) 175; also in Field Theory, Quantum Gravity and Strings, eds. H.J. de Vega and N. Sanchez (Srpinger, Berlin 1986).

[4] F. Lombardo and F. D. Mazzitelli, Phys. Rev. D 53 (1996) 2001 [arXiv:hep-th/9508052]; Phys. Rev. D 58 (1998) 024009 [arXiv:gr-qc/9712091]; E. Calzetta and B. L. Hu, Phys. Rev. D 52 (1995) 6770 [arXiv:gr-qc/9505046]; E. A. Calzetta, B. L. Hu, and F. D. Mazzitelli, Phys. Rept. 352 (2001) 459 [arXiv:hep-th/0102199], and references therein. C. Kiefer, "Quantum gravity," (Oxford, UK: Clarendon (2004), International series of monographs on physics. 124, and references therein.

[5] F. C. Lombardo, R. J. Rivers, and F. D. Mazzitelli, Int. J. Theor. Phys. 41, 2121 (2002) [arXiv:hep-ph/0202042]; Int. J. Theor. Phys. 41, 2145 (2002) [arXiv:hep-ph/0202041]. F. C. Lombardo, F. D. Mazzitelli, and R. J. Rivers, Phys. Lett. B 523, 317 (2001) [arXiv:hep-ph/0102152].

[6] S. Hellerman, N. Kaloper, and L. Susskind, JHEP 0106, 003 (2001) [arXiv:hep-th/0104180]; W. Fischler, A. Kashani-Poor, R. McNees, and S. Paban, JHEP 0107, 003 (2001) [arXiv:hepth/0104181]; J. R. Ellis, N. E. Mavromatos, and D. V. Nanopoulos, arXiv:hep-th/0105206; E. Witten, arXiv:hep-th/0106109; T. Banks and M. Dine, JHEP 0110, 012 (2001) [arXiv:hepth/0106276]; P. O. Mazur and E. Mottola, Phys. Rev. D 64, 104022 (2001) [arXiv:hep-th/0106151].

[7] W. Fischler and L. Susskind, Phys. Lett. B 173, 262 (1986);
Phys. Lett. B 171, 383 (1986).

[8] F. David, Mod. Phys. Lett. A 3, 1651 (1988); J. Distler and H. Kawai, Nucl. Phys. B 321, 509 (1989); J. Distler, Z. Hlousek, and H. Kawai, Int. J. Mod. Phys. A 5, 391 (1990); N. E. Mavromatos and J. L. Miramontes, Mod. Phys. Lett. A 4, 1847 (1989); E. D'Hoker and P. S. Kurzepa, Mod. Phys. Lett. A 5, 1411 (1990).

[9] J. R. Ellis, N. E. Mavromatos, and D. V. Nanopoulos, Phys. Lett. B 293, 37 (1992) [arXiv:hep-th/9207103]; Invited review for the special Issue of J. Chaos Solitons Fractals, Vol. 10, (eds. C. Castro amd M.S. El Naschie, Elsevier Science, Pergamon 1999) 345 [arXiv:hep-th/9805120]; Phys. Rev. D 63, 024024 (2001) [arXiv:gr-qc/0007044].

[10] E. Gravanis and N. E. Mavromatos, Phys. Lett. B 547, 117 (2002) [arXiv:hep-th/0205298].

[11] N. E. Mavromatos, arXiv:hep-ph/0309221; arXiv:hep$\mathrm{ph} / 0402005$.

[12] R. Wald, Phys. Rev. D 21, 2742 (1980).

[13] G. Barenboim and N. E. Mavromatos, JHEP 0501, 034 (2005) [arXiv:hep-ph/0404014].

[14] G. Barenboim and N. E. Mavromatos, Phys. Rev. D 70, 093015 (2004) [arXiv:hep-ph/0406035].

[15] L. Wolfenstein, Phys. Rev. D 17, 2369 (1978); S. P. Mikheev and A. Y. Smirnov, Sov. J. Nucl. Phys. 42, 913 (1985) [Yad. Fiz. 42, 1441 (1985)].

[16] M. Blasone and G. Vitiello, Annals Phys. 244, 283 (1995) [Erratum-ibid. 249, 363 (1996)] [arXiv:hep-ph/9501263];

[17] M. Blasone, A. Capolupo, S. Capozziello, S. Carloni, and G. Vitiello, Phys. Lett. A 323, 182 (2004) [arXiv:grqc/0402013].

[18] G. Lindblad, Commun. Math. Phys. 48, 119 (1976). R. Alicki and K. Lendi, Lect. Notes Phys. 286, Springer-Verlag, Berlin 
(1987).

[19] J. Ellis, J. Hagelin, D.V. Nanopoulos, and M. Srednicki, Nucl. Phys. B 241, 381 (1984).

[20] J. R. Ellis, N. E. Mavromatos, and D. V. Nanopoulos, Phys. Lett. B 293, 142 (1992) [arXiv:hep-ph/9207268]; J. R. Ellis, J. L. Lopez, N. E. Mavromatos, and D. V. Nanopoulos, Phys. Rev. D 53, 3846 (1996) [arXiv:hep-ph/9505340]; P. Huet and M. E. Peskin, Nucl. Phys. B 434, 3 (1995) [arXiv:hep$\mathrm{ph} / 9403257]$;

[21] E. Lisi, A. Marrone, and D. Montanino, Phys. Rev. Lett. 85, 1166 (2000) [arXiv:hep-ph/0002053].

[22] F. Benatti and R. Floreanini, JHEP 0002, 032 (2000) [arXiv:hep-ph/0002221]; Phys. Rev. D 64, 085015 (2001) [arXiv:hep-ph/0105303].

[23] F. Benatti and R. Floreanini, Phys. Lett. B 468, 287 (1999) [arXiv:hep-ph/9910508].

[24] A. M. Gago, E. M. Santos, W. J. C. Teves, and R. Zukanovich Funchal, arXiv:hep-ph/0208166.

[25] A. Aguilar et al. [LSND Collaboration], Phys. Rev. D 64, 112007 (2001) [arXiv:hep-ex/0104049]; G. Drexlin, Nucl. Phys. Proc. Suppl. 118, 146 (2003).

[26] M. Apollonio et al. [CHOOZ Collaboration], Phys. Lett. B 466, 415 (1999) [arXiv:hep-ex/9907037].

[27] M. Jacobson and T. Ohlsson, Phys. Rev. D 69, 013003 (2004) [arXiv:hep-ph/0305064]

[28] T. Ohlsson, Phys. Lett. B 502, 159 (2001) [arXiv:hep$\mathrm{ph} / 0012272]$.

[29] K. Eguchi et al. [KamLAND Collaboration], Phys. Rev. Lett. 90, 021802 (2003) [arXiv:hep-ex/0212021].

[30] B. Armbruster et al. [KARMEN Collaboration], Phys. Rev. D 65, 112001 (2002) [arXiv:hep-ex/0203021].

[31] T. Alexopoulos et al. [KTeV Collaboration], arXiv:hep- ex/0406002.

[32] E. D. Zimmerman [BooNE Collaboration], eConf C0209101, TH05 (2002) [Nucl. Phys. Proc. Suppl. 123, 267 (2003)] [arXiv:hep-ex/0211039].

[33] K. Lang, Int. J. Mod. Phys. A 18, 3857 (2003).

[34] see for instance: D. Hooper, D. Morgan, and E. Winstanley, arXiv:hep-ph/0410094, and references therein.

[35] H. b. Zhang, Z. j. Cao, and C. s. Gao, Commun. Theor. Phys. 41 (2004) 385 [arXiv:gr-qc/0308064]; G. Lifschytz, arXiv:hepth/0405042.

[36] F. N. Loreti and A. B. Balantekin, Phys. Rev. D 50, 4762 (1994); E. Torrente-Lujan, arXiv:hep-ph/0210037.

[37] J. R. Ellis, N. E. Mavromatos, and M. Westmuckett, Phys. Rev. D 70, 044036 (2004) [arXiv:gr-qc/0405066].

[38] L.P. Houghston, Proc. Roy. Soc. London A 452, 953 (1996); I.C. Percival, Proc. Roy. Soc. London A 447, 189 (1994); S. L. Adler, Phys. Rev. D 62, 117901 (2000).

[39] S. M. Bilenky and B. Pontecorvo, Phys. Rept. 41, 225 (1978).

[40] K. C. Hannabuss and D. C. Latimer, J. Phys. A 33, 1369 (2000); ibid. 36, L69 (2003).

[41] M. Blasone, P. A. Henning, and G. Vitiello, Phys. Lett. B 451, 140 (1999).

[42] C. Giunti, arXiv:hep-ph/0409230, and references therein.

[43] M. Blasone, J. Magueijo, and P. Pires-Pacheco, arXiv:hep$\mathrm{ph} / 0307205$.

[44] G. Amelino-Camelia, Int. J. Mod. Phys. D 11, 35 (2002) [arXiv:gr-qc/0012051]; J. Magueijo and L. Smolin, Phys. Rev. D 67, 044017 (2003) [arXiv:gr-qc/0207085].

[45] G. Amelino-Camelia, L. Smolin, and A. Starodubtsev, 21, 3095 (2004) [arXiv:hep-th/0306134], and references therein. 